\title{
Ambientes Virtuales de Aprendizaje: dificultades de uso en los estudiantes de cuarto grado de primaria ${ }^{1}$ Environments of virtual learning: difficulties of use faced by students of fourth grade
}

\author{
Adrián Filiberto Contreras-Colmenares* \\ Luz Myla Garcés-Díaz ${ }^{* *}$
}

\section{Resumen}

Los Ambientes Virtuales de Aprendizaje son entendidos como los espacios o entornos que favorecen el aprendizaje de los alumnos, con apoyo en la tecnología, mediante la cual se genera una interactuación entre los discentes y el docente. Su uso, generalmente presenta dificultades por parte de los estudiantes de cuarto grado. Y esos impedimentos que impactan en el aprendizaje han sido un asunto de estudio constante, porque representan un escollo en el proceso formativo del ser humano. De ahí que se haya planteado como propósito analizar las dificultades que presentan los estudiantes de cuarto grado de primaria para aprender en, con y desde la virtualidad. Como se ha documentado en diferentes estudios, las Tecnologías de la Información y la Comunicación (TIC) han venido transformando la educación, puesto que, como recurso didáctico, plantean nuevos escenarios, que requieren una revisión profunda del contexto

${ }^{1}$ Artículo que se deriva de la investigación Dificultades que presentan los estudiantes de cuarto grado de primaria al trabajar en Ambientes Virtuales de Aprendizaje específicamente en la plataforma Chamilo, desarrollada en el período 2012-2013. Artículo que es avalado por el Centro de Investigación “Georgina Calderón”, adscrito a la Universidad Pedagógica Experimental Libertador, Venezuela.

* Doctor en Educación, Abogado. Docente Emérito. Profesor Jubilado, categoría Titular de la Universidad de Los Andes. Táchira, Venezuela. Correo electrónico: adriancontreras@ ula.ve

${ }^{* *}$ Licenciada en Educación Básica. Maestra en Tecnología educativa. Universidad Virtual Escuela de Graduados en Educación. Instituto Tecnológico de Monterrey. México. Magistra en Tecnología Educativa y Medios Innovadores para la Educación. Universidad Autónoma de Bucaramanga. Docente de primaria de la Institución Educativa Provenza. Bucaramanga, Colombia. Correo electrónico: luzmi0404_garcesdiaz@hotmail.com

Universidad del Valle. Cali, Colombia. Recibido: 30/07/2018 Aprobado: 10/11/201 $\overline{8}$

ISSN: 0122-1213 ISSN-e: 2389-993X. Doi: 10.25100/prts.v0i27.7273 
educativo. Se utilizó un enfoque mixto en el análisis de la información y se sustentó en un estudio de carácter descriptivo. Se llegó, entre otras, a la conclusión siguiente: Es importante realizar una transformación de la práctica educativa mediante la incorporación de las TIC en el contexto educativo, como fundamento del Ambiente Virtual de Aprendizaje (AVA).

Palabras clave: Educación básica primaria; Ambientes virtuales de aprendizaje; TIC; Dificultades aprendizaje; Práctica educativa.

\section{Abstract}

Virtual Learning Environments are understood as spaces or environments which favor learning with the support of technology, and through which interaction between the learner and the teacher is generated. Fourth grade students generally face difficulties in such learning environments, and the impediments that impact learning have been a matter of constant study, because they represent a stumbling block in the formative process of the human being. Hence, the purpose of this study is to analyze the difficulties encountered by students in the fourth grade of primary school to learn within, with and from virtuality. The state-of-the-art shows that Information and Communication Technologies (ICT) have been transforming education, since, as a didactic resource, they propose new scenarios, which require a profound revision of the educational context. A mixed approach was used in the analysis of the information and was based on a descriptive study. The following conclusion, among others, was reached: It is important to transform the educational practice by incorporating ICT in the educational context, as the foundation of the Virtual Learning Environment (AVA).

Keywords: Primary basic education; Virtual learning environments; ICT; Learning difficulties; Educational practice.

Sumario: 1. Introducción; 2. Aspectos teóricos; 3. Metodología; 3.1. Escenario de investigación y los informantes; 3.2. Técnicas e instrumentos de recolección de información; 4. Hallazgos; 5. Conclusiones; 6. Referencias bibliográficas. 


\section{Introducción}

Afrontar el aprendizaje por parte de los alumnos, cada día se hace más complejo, por las múltiples variables intervinientes, tanto en el espacio educativo, como en el contexto social. La complejidad, en el ámbito escolar, se debe a varios factores. Unos factores están asociados a las capacidades propias de los estudiantes en cuanto a lo cognitivo, los intereses y motivación por el aprendizaje; aspectos que no forman parte de la temática de esta elaboración discursiva. Otros factores, dentro del espacio escolar, están asociados con el uso de estrategias, técnicas, métodos y recursos empleados por los docentes.

Dentro de los recursos, se asumen lo referido a la incorporación, en el proceso de aprendizaje, de las Tecnologías de la Información y la Comunicación (TIC) y, dentro de ellas, los entornos que, de manera asíncrona o también sincrónica, ayudan, mediante la interacción entre estudiantes y docentes, a fortalecer el aprendizaje de las diferentes áreas del conocimiento. A estos entornos se les denominan Ambientes Virtuales de Aprendizaje (AVA), y forman parte de las distintas plataformas tecnológicas con que pudiere contar una institución escolar o también en plataformas tecnológicas de otras instituciones. A través de los AVA, como concreción de las TIC, se puede promover el aprendizaje significativo de los estudiantes del nivel primario, y ese tópico es el foco de atención de este reporte investigativo.

Así entonces, en lo que respecta a la incorporación de las TIC en el nivel primario, se ha encontrado el trabajo de Castañeda-Castañeda, CarriloÁlvarez y Quintero-Monreal (2013) quienes indican

...hablar del uso de las TIC en educación primaria a partir de la experiencia con Enciclomedia como herramienta pedagógica y nos obliga a preguntarnos ¿qué pasó con este programa educativo? ¿Dónde quedó el impulso ofrecido por el gobierno federal al promover la implementación de un modelo pedagógico tecnológico, operativo, de gestión escolar y de acompañamiento a los docentes, que sirviera para desarrollar las competencias de los alumnos y favorecer su inserción en la sociedad del conocimiento? (p. 6). 
Como se lee, hay herramientas pedagógicas, que se pueden incluir para apoyar el aprendizaje de los alumnos de educación primaria, por parte de quienes se encuentran con la responsabilidad de orientar, guiar o dirigir los procesos de enseñanza y aprendizaje. Y una de esas herramientas pedagógicas es el uso de los computadores u ordenadores, como recursos tecnológicos que coadyuvan en el proceso de aprendizaje de los estudiantes de educación primaria.

Por su parte, Romera-Blasco (2014), en otra investigación realizada, expresa que: ... "la educación primaria debe facilitar la preparación en competencias digitales para ayudar a los niños a desenvolverse en esta sociedad" (p. 15). De modo que, en esa preparación y a través de las TIC, los estudiantes de primaria van accediendo a los conocimientos que se producen en las comunidades académicas y en la sociedad del conocimiento.

En consonancia con este planteamiento, Cebrián de la Serna (2003) manifiesta: ... "las nuevas tecnologías de la Información y la Comunicación [están] asociadas a la producción del conocimiento y a los procesos de innovación educativa” (p. 16). Así, las TIC han venido influyendo en el ámbito educativo y han logrado transformar la cultura del aprendizaje y la enseñanza; por tanto, surge la necesidad de implementar modelos educativos innovadores para fomentar ambientes de aprendizaje significativos, en el que el docente se comprometa a dejar atrás los paradigmas convencionales o clásicos de enseñanza.

El docente, por tanto, para adaptarse a las nuevas circunstancias de enseñanza y de mediación instrumental, propuesta por Vygotsky (en Contreras-Colmenares, 2004), tiene que asumir el dominio de las nuevas tendencias tecnológicas y habrá de incorporar las TIC en el aula de clase, para promover un aprendizaje significativo en los estudiantes de primaria. De ese modo, con apoyo en las TIC, podrá descubrir la significación del trabajo docente en AVA, y de la actividad que han de cumplir los estudiantes del nivel primario. Los AVA forman parte de la educación virtual y, en ese tenor, Rubio-Moraga (2003) expresa: "[La educación virtual] es un proceso de formación de carácter auto dirigido, en donde el mismo estudiante avanza según sus intereses, capacidad de auto-aprendizaje y habilidades para el autoestudio" (p. 5). 
En función de lo propuesto, y a fin de que se pueda lograr un avance progresivo en el aprendizaje de los estudiantes de primaria, se hace necesario que los docentes potencien los Ambientes Virtuales de Aprendizaje con la inclusión de actividades pedagógicas, que promuevan el desarrollo de habilidades en los estudiantes para que se pueda romper con la brecha digital -en cuanto que su no dominio se toma como criterio de desigualdad- lo cual afecta tanto a las instituciones educativas, como a los usuarios que, en este caso de estudio, son los estudiantes de primaria del grado cuarto.

Así entonces, hay que aprovechar al máximo las condiciones favorables de los AVA y, por supuesto, lo efectivas que pueden ser las TIC para el aprendizaje significativo de los estudiantes. Una revelación de la efectividad de las TIC en la educación primaria, por su permanente actualización y organización, la hace Arias-Arranz (2017), quien, a partir de la investigación realizada, indicó:

El CRA [Centro de Recursos para el Aprendizaje] los Almendros cuenta con certificado TIC nivel 5. Acceso a internet a través de ADSL. La página web se actualiza diariamente siendo muy completa su información y bien organizada. En Ciencias Naturales y Ciencias Sociales de $5^{\circ}$ y $6^{\circ}$ de Educación Primaria trabajan con contenido digital no necesitando libro de texto. Se accede al material a través de la intranet del centro para lo que cuenta con un disco duro a modo de servidor (p. 33).

Así las cosas, las TIC incorporadas como apoyo del aprendizaje son de alto significado y han de estar en constante actualización, y estar disponibles a cualquier hora en que sea requerido su uso, tanto por el docente, como por sus estudiantes. De ahí que un recurso que puede utilizarse son los AVA, los cuales se constituyen en excelentes campos de la virtualidad, entornos o espacios físicos tecnológicos, que, a su vez, favorecen el aprendizaje y el intercambio académico, así como la transacción del conocimiento entre docente y alumnos.

De ese modo, se fortalecerán tanto el proceso de enseñanza, como el proceso de aprendizaje. En correspondencia con esta idea Gros-Salvat (2011) afirma: 
Existen varios elementos que marcan el proceso de aprendizaje de (...) [cada] estudiante y que hay que tener en cuenta a la hora de analizar su situación: inquietudes, aproximación a una institución educativa, elección, formalización del compromiso, expectativas, aprendizaje de las distintas materias, certificación de aptitud, finalización de los estudios, graduación (p. 28).

Estos presupuestos esgrimidos por Gros-Salvat (2014) permiten revelar la importancia que tienen los entornos virtuales de aprendizaje, para que el alumno de primaria desarrolle su proceso constructivo relacionado con el conocimiento (el aprendizaje de los dominios del conocimiento).

No obstante, en la realidad se ha podido percibir que los recursos tecnológicos, específicamente los AVA, no han sido utilizados quizá por cierto desconocimiento, por un lado, por parte de los docentes, acerca de los requerimientos establecidos para su incorporación como parte de esa relación pedagógica: docente-alumno-entornos virtuales de aprendizaje. Y, por otro lado, por parte de los estudiantes, quienes tienen las dificultades propias de acceso a las tecnologías, aun cuando, a esta generación se le conoce como migrantes digitales.

Empero, la razón de mayor peso, en los estudiantes de grado cuarto de primaria es que, en su mayoría, residen en zonas rurales, y, por tanto, no cuentan con el servicio de internet para poder apoyar su aprendizaje. Sus padres de escasos recursos económicos, no pueden tampoco proveerles de equipos tecnológicos, con los cuales puedan relacionarse con los AVA. Solamente, tienen contacto con estos recursos tecnológicos en la institución educativa y, por muy poco tiempo, por la restricción del horario.

Hay que afirmar que en el Colegio Integrado Nuestra Señora de la Paz, hay una sala de informática con internet, bajo la plataforma Chamilo, que permite organizar procesos de enseñanza aprendizaje a través del trabajo individual o colaborativo. Desafortunadamente, pocos docentes y pocas veces se usa para el desarrollo de actividades de aprendizaje. Este poco uso se debe quizá a que varios docentes, en cierto modo, pareciera que ignoran cuáles son los beneficios que se obtienen cuando se incluyen estrategias mediadas por las TIC. 
Además, porque los estudiantes presentan dificultades para trabajar con los computadores y realizar la exploración en internet. A ello se aúna que cuando los alumnos se encuentran con la tecnología, lo quieren utilizar para jugar y no para buscar información que les permita fortalecer su aprendizaje en determinadas áreas.

Dado que pocos docentes lo utilizan, la actividad investigativa se realizó con el apoyo de la docente de grado cuarto, quien, a su vez, fungió como investigadora principal, en su condición de observadora participante. Los actos pedagógicos fueron realizados en la sala de informática y allí se hicieron las observaciones.

Necesario informar que cuando los alumnos se encuentran en la sala de informática, de acuerdo con las observaciones realizadas, el docente propone unas asignaciones, a modo de preguntas, para ser respondidas con apoyo en las TIC. De ese modo, consideran que pueden fortalecer y consolidar un aprendizaje significativo de los contenidos curriculares desarrollados. En conclusión, el encuentro con los AVA por parte de los alumnos y los docentes participantes en la investigación se da en la institución, dentro de la sala de informática.

Como se ha explicado, se planteó como intención investigativa la indagatoria acerca de las dificultades que presentan los estudiantes de cuarto grado de primaria, en cuanto al uso de los AVA, que, consecuencialmente, impacta en el aprendizaje significativo. En ese sentido, para realizar la investigación se seleccionó intencionalmente a 20 estudiantes del grado cuarto de primaria. Para la recolección de la información se utilizaron como técnicas la encuesta y la observación y como instrumentos, el cuestionario - con preguntas abiertas y cerradas-y la rejilla de observación respectivamente, con criterios establecidos que se incluyen en el apartado correspondiente a las técnicas e instrumentos de investigación.

En lo que respecta al análisis de la información recabada, tanto por la aplicación del cuestionario, como lo registrado en la rejilla de observación, se utilizó el enfoque mixto (apoyo en fundamentos cualitativos y cuantitativos), de modo que los datos fueron categorizados y comparados entre sí. Además, se establecieron cuatro categorías para realizar el agrupamiento de la información. Estas categorías fueron elaboradas 
así: (a) habilidades comunicativas, (b) habilidades interpretativas, (c) autoaprendizaje y (d) habilidades computacionales, cuya descripción se presenta en el apartado correspondiente al análisis e interpretación.

\section{Aspectos teóricos}

Una condición que da cuenta del progreso académico es la que permite saber que se está en sintonía con los cambios que se van produciendo tanto en los espacios concretos de enseñanza y de aprendizaje, como en los espacios virtuales, a partir de la aparición de la tecnología y de su constante evolución. Hoy, con mucha frecuencia se escribe y se discute, en los diferentes eventos académicos, acerca de términos vinculados con lo tecnológico para la mejora y fortalecimiento de los aprendizajes de los estudiantes.

Así surgen, los Ambientes Virtuales de Aprendizaje:

El concepto "ambientes virtuales de aprendizaje" (AVA) nace casi de la mano con la utilización del adjetivo "virtual", y el cual está referido a las organizaciones, comunidades, actividades y prácticas que operan y tienen lugar en Internet; y se subraya su potencialidad por permitir una comunicación entre usuarios, similar a la que se realiza cara a cara (Coll y Monereo citados por Valencia-Vallejo, Huertas-Bustos y BaracaldoRamírez, 2014, p. 80).

De la información precedente se puntualiza en lo siguiente: (a) los ambientes virtuales de aprendizaje, son coetáneos en su esencia originaria con la adjetivación virtual, (b) la virtualidad se asocia con todos los eventos, circunstancias y actores que tienen su acción en Internet y (c) se plantea la robustez que se da en el proceso comunicacional entre los internautas, que se asemeja a lo que ocurre en la interactuación presencial, sólo que ese proceso comunicacional está caracterizado, esencialmente, por la asincronía y la usabilidad.

Necesario, señalar que muchos autores han optado también por denominar a los ambientes virtuales de aprendizaje, como entornos virtuales de aprendizaje. Así por ejemplo Adell, Castellet \& Pascual (citados por Silva, 2017) indican: 
Un entorno virtual de aprendizaje (EVA) es una aplicación informática diseñada para facilitar la comunicación pedagógica entre los participantes en un proceso educativo, sea éste completamente a distancia, presencial, o de una naturaleza mixta que combine ambas modalidades en diversas proporciones (p. 4).

Hay coincidencia en la descripción y en su uso, sea que se denominen AVA o EVA, por cuanto la esencia se centra en que procuran la comunicación entre participantes o plurales usuarios, con la intención variada de recrearse, de cultivarse académicamente o de construir aprendizajes. Importante, resaltar que en la precisión definicional presentada se revela la comunicación pedagógica, como parte del entramado relacional entre los diferentes actores que se vinculan mediante el proceso educativo.

Como se puede valorar a continuación, los AVA, en la visión de Dillenbourg, Schneider y Synteta (citados por Valencia-Vallejo, HuertasBustos y Baracaldo-Ramírez, 2014) son definidos de la siguiente manera:

...como un espacio de información diseñado para un proceso educativo, en donde se comunican los actores que intervienen en él de manera efectiva y constante, obedeciendo a unos principios pedagógicos que orientan el desarrollo de las temáticas establecidas para el aprendizaje (p. 80).

Se reafirma la particularidad de considerarlos como un espacio con carácter informativo, pero, se hace necesario, afirmar que ha de trascender ese carácter informacional, para erigirse en un punto de reflexión y de interactuación entre los distintos elementos del currículo que intervienen en el acto predagógico de modo virtual, pero con la intención de avanzar hacia un aprendizaje significativo. Este presupuesto cobra particular importancia, por cuanto se hace uso de la tecnología y de todos los recursos que se encuentran a disposción de los internautas.

En esa intención, Ospina-Pineda (2014) expresa:

Podemos decir que un ambiente virtual de aprendizaje es un entorno de aprendizaje mediado por tecnología que transforma la relación educativa gracias a: la facilidad de comunicación y procesamiento, la gestión y la distribución de información, agregando a la relación educativa 
nuevas posibilidades y limitaciones para el aprendizaje. Los ambientes o entornos virtuales de aprendizaje son instrumentos de mediación que posibilitan las interacciones entre los sujetos y median la relación de estos con el conocimiento, con el mundo, con los hombres y consigo mismo.

Las tecnologías de la información y la comunicación aplicadas a la educación y a la creación de entornos virtuales de aprendizaje nos dan la posibilidad de romper las barreras espacio temporales que existen en las aulas tradicionales y posibilitan una interacción abierta a las dinámicas del mundo (p. 1).

Se hace concreción en los siguientes elementos: (a) hay una mediación tecnológica. Este criterio se puede vincular con lo expresado por Vygostky (en Contreras-Colmenares, 2004) acerca de la mediación instrumental en los siguientes términos: "Los seres humanos (...) con frecuencia hacen uso de variados instrumentos y signos que les permiten orientar su atención, sistematizar u organizar su memoria consciente y tener un control sobre su conducta" (pp. 183-184); (b) existe llaneza en el proceso comunicacional, así como en su procesamiento, entre otras acciones, que, indubitablemente, van a generar posibilidades nuevas de enfrentar el aprendizaje, aun cuando, no se desdeña la emergencia de limitaciones.

Otras desagregaciones de la idea planteada por Ospina-Pineda (2014) son las siguientes: (c) se ratifica que los AVA-EVA son una instrumentalidad que favorece la mediación. Entonces, hay que comprender que la mediación consiste en la relación entre un sujeto y un objeto. Ese ligamen está articulado como lo refiere Cole (Daniels, 2003) a través de un "artefacto"; esto es, un instrumento disponible culturalmente. Por tanto, el sujeto se aproxima, llega o accede al objeto mediante un instrumento; vale argüir, a través del artefacto, según Cole. Por tanto, imprescindible entender que en ese proceso relacional, el sujeto es quien juega un papel muy activo en aras de su propio desarrollo, autoaprendizaje o autoconstrucción.

Ello, en razón de que como afirma Daniels (2003): ..."el ser humano tiene un proceso de (...) 'autoconstrucción mediante los instrumentos que están disponibles"” (p. 67). Por tanto, se hace evidente la necesidad de la presencia de la instrumentalidad para construir los conocimientos; en este caso, los AVA-EVA se convierten en el "artefacto", en el instrumento 
que permite, por un lado, la comunicación entre diversos actores, asíncronamente, y, por el otro, favorece el aprendizaje significativo y útil.

Ese acto constructivo, a su vez, va a tener como refente los sistemas simbólicos culturales. Ello se logra en los AVA-EVA, mediante los cuales los actores de los procesos comunicacionales van a estructurar su proceso de realización y de construcción. Es pertinente, por tanto, atender lo planteado por Vygotsky (Daniels, 2003) en los siguientes términos:

...los seres humanos se dominan a sí mismos mediante sistemas simbólicos culturales externos en lugar de estar subyugados por ellos. Puesto que este estímulo auxiliar posee la función específica de la acción inversa, transfiere la actividad psicológica a formas superiores, y cualitativamente nuevas, y permite que los seres humanos, mediante la ayuda de estímulos extrínsecos, controlen su conducta desde el exterior (p. 33).

Si valoramos que, en los AVA, la tecnología se convierte en un instrumento auxiliar para el aprendizaje y para la enseñanza, entonces, se puede colegir que en la acción que realiza el aprendiz, una vez que entra es cuando se produce una acción intelectiva o "actividad psicológica" traducida en aprendizaje. Ello se logrará mediante la activación de procesos cognitivos superiores que le van a posibilitar el desarrollo de otro tipo de aprendizaje mucho más preciso, característico y propio, con fundamento en la instrumentalidad.

\section{Metodología}

La investigación se desarrolló mediante un estudio descriptivo; en ese sentido, Danhke (citado por Hernández, Fernández y Baptista, 1994) sostiene: "Los estudios descriptivos buscan especificar las propiedades, las características y los perfiles de las personas, grupos, comunidades, procesos, objetos o cualquier otro fenómeno que sea sometido a análisis" (p. 60). Y, en esta perspectiva de investigación, tal como manifiesta García-

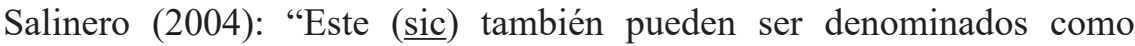
estudios transversales, de corte, de prevalencia" (p. 1). 
Así entonces, por un lado, a través de los estudios descriptivos se pueden hacer caracterizaciones, pero también mediciones, una vez que se haya recolectado la información, y esas mediciones permitirán realizar individualizaciones, del fenómeno estudiado. Por el otro lado, en cuanto son estudios transversales o sincrónicos, quiere indicar que se aplican en un solo momento y a un mismo grupo de informantes o de estudio. Tal como se hizo en esta investigación.

Se indica, además, que para el análisis se ha optado por una mixtura entre la vertiente cualitativa y la vertiente cuantitativa. Ello, en atención, al planteamiento que hiciesen Villar y Marcelo (1992) en los siguientes términos:

...los progresos de la investigación en el ámbito de las ciencias sociales resultan de aplicar combinaciones explícitas de métodos, o sea una multitud de métodos dependientes de las preguntas de investigación, y no de limitar el problema y los accesos empíricos según las posibilidades de un enfoque metodológico determinado (p. 186).

Ahora bien, necesario referir que se ha incorporado el enfoque mixto en lo que tiene que ver con el procedimiento de análisis e interpretación de los resultados y no en cuanto al paradigma epistemológico. En razón de ello, se ha incluido el análisis descriptivo, vinculado a la estadística descriptiva, en cuanto se presentan las frecuencias y los porcentajes, de los datos recabados en las encuestas. Y en la visión cualitativa se han creado categorías, en las que se incluye lo hallado en las observaciones. Entonces, en función de las categorías se integran tanto los datos cuantitativos (porcentajes) como la información producto de lo observado.

Ello, en razón de lo que exponen Creswell y Tashakkori (en Hernández, Fernández y Baptista, 2010): “[En] El reporte [de la investigación] tienen que incluirse ambas aproximaciones en la recolección, análisis e integración de datos" (p. 592).

\subsection{Escenario de investigación y los informantes}

Para el caso de nuestra investigación se tomó como centro de atención indagatoria el Colegio Integrado Nuestra Señora de la Paz, ubicado en el Prospectiva. Revista de Trabajo Social e intervención social No. 27, enero-junio 2019: pp. 215-240 @) () () 
Municipio de Betulia, Santander, Colombia. Y los estudiantes seleccionados fueron veinte (20), quienes se encontraban cursando el cuarto grado, cuyas edades estaban comprendidas entre 9 y 10 años. La selección se hizo de manera intencional en función del objetivo planteado y por cuanto, a través de la plataforma Chamilo, se está trabajando con los AVA.

\subsection{Técnicas e instrumentos de recolección de información}

A los efectos de recabar la información se optó por utilizar la técnica de la observación y la técnica de la encuesta. De la técnica de la observación se utilizó una rejilla de observación de clase, que puede ser definida como: El recurso real que permite guiar el proceso de observación que realiza un investigador-observador, a un aspecto principal de la actividad que se desarrolla en el aula de clase. Un aspecto esencial, que se valora como centro de atención del investigador y que con el propósito de precisarlo formula criterios observacionales.

En la literatura se encuentra bajo la denominación de parrilla de observación o, sencillamente, rejilla de observación. Y es, unas veces, valorada como técnica y otras veces como instrumento. Desde esta premisa, Leiva-Zea (2010) la define así: "La rejilla de observación es una técnica que consiste en observar atentamente el fenómeno, hecho o caso, tomar información y registrarla para su posterior análisis".

En esa perspectiva, se debe señalar que la rejilla de observación de clase, permite registrar los aspectos observados; por tanto, el investigador puede adoptar una posición flexible frente al fenómeno y tiene la gran ventaja de su adaptabilidad frente a sucesos inesperados.

El diseño de la rejilla de observación de clase se diseñó de conformidad con los siguientes criterios: (a) redacción y escritura de textos, (b) comunicación y escucha, (c) intercambio de ideas, (d) desarrollo de actividades, (e) comprensión lectora, (f) navegación en la plataforma, (g) solución a dificultades presentadas, (h) trabajo individual (i) manejo y conocimiento del computador.

La observación se desarrolló durante dos sesiones de trabajo, número que permitió registrar los hechos relevantes en función de cada criterio. 
El número de observaciones se valoró como suficiente, en función de la intención indagatoria establecida.

Con respecto a la recolección de datos mediante la técnica de la encuesta, se aplicó un cuestionario estructurado con una serie de interrogantes abiertos y cerrados, cuya estructura se hizo teniendo en cuenta las habilidades desarrolladas en el trabajo realizado con el apoyo de los AVA, esencialmente, bajo la plataforma Chamilo, y se formularon trece (13) preguntas con opciones de respuestas cerradas y tres (3) preguntas con opciones de respuestas abiertas. Las preguntas se elaboraron en función de determinar: (a) las habilidades y competencias tecnológicas, (b) habilidades comunicativas, (c) habilidades interpretativas, (d) interactuación con sus coetáneos y con el docente, y (e) el proceso de autoaprendizaje.

\section{Procedimiento de recolección de la información}

Para recabar la información, se desarrolló mediante una serie de fases o etapas:

1. Entrada al escenario (lugar donde se encuentran los equipos tecnológicos)

2. Se analizó el grupo de estudiantes, y se seleccionó intencionalmente a los alumnos de cuarto grado.

3. Con respeto a los dictámenes éticos de la investigación, se comunicó a los estudiantes y a sus padres la naturaleza y objetivo de la investigación antes de iniciarla a través de la carta de participación.

4. Se realizó la observación cuando los estudiantes participaron en actividades en la clase de español, al momento de desarrollar la temática sobre comprensión lectora, y cuando hacían uso de un ambiente virtual de aprendizaje; para ello, se utilizó la rejilla de observación de clase. Cada actividad tuvo una duración de 2 horas.

5. Una vez concluido el proceso de observación, se aplicó un cuestionario en un formato predeterminado con preguntas cerradas y abiertas, con el fin de conocer la opinión de los estudiantes sobre las habilidades aplicadas en la actividad desarrollada en el ambiente virtual de aprendizaje. 
6. Se depuró la información recolectada y se eliminó la información que no fue útil para responder a la pregunta de investigación planteada.

7. Se organizó y tabuló la información.

8. Se analizó la información recogida.

9. Se elaboró el informe de la investigación

\section{Hallazgos}

La organización de presentación de la información obtenida, mediante el cuestionario y la rejilla de observación de clase se hizo con base en cuatro (04) categorías, que fueron elaboradas a partir de lo recabado. Dichas categorías fueron las siguientes: (a) habilidades comunicativas, (b) habilidades interpretativas, (c) autoaprendizaje y (d) habilidades computacionales. Los hallazgos se codificaron y se analizaron de manera conjunta (datos obtenidos por la aplicación del cuestionario e información proporcionada mediante la observación, a través del uso de la rejilla de observación de clase) de forma tal que permitieron tener una comprensión más fiel y fidedigna del fenómeno estudiado, mediante la comparabilidad informativa ${ }^{2}$.

\section{Categoría habilidades comunicativas.}

Los datos arrojados permiten indicar que el $75 \%$ de los estudiantes tienen dificultad para la expresión de sus ideas, pensamientos y deseos, a través del lenguaje. Los estudiantes presentan dificultades para expresar sus ideas, interactuar con sus compañeros, comprender mensajes, leer y escribir; estas falencias se registraron en la observación y se reafirman en las respuestas dadas por los estudiantes en el cuestionario, donde el $65 \%$ reconoce no haber utilizado un lenguaje, tanto oral como escrito en una forma clara y coherente.

La información contenida en esta descripción se presenta en la Figura 1:

${ }^{2}$ Expresión propuesta por los autores, como vía para ir estructurando un léxico más cercano a la realidad que viven los maestros y los investigadores nacionales. 
Figura 1. Categoría: Competencias comunicativas

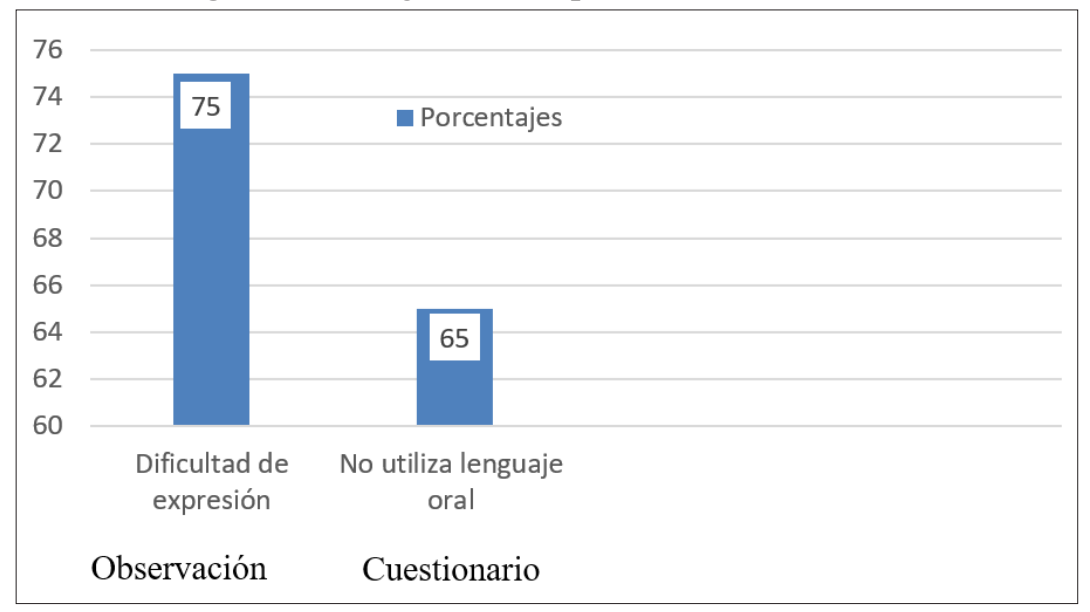

Fuente: elaboración propia

De lo observado en el decurso de la investigación y de lo respondido por los estudiantes de primaria, puede señalarse que estos inconvenientes, relacionados con las competencias comunicativas (caracterizadas, a veces, por la poca interactuación con sus pares y con el docente), se presentan quizás por lo limitado del tiempo para estar en contacto con los equipos tecnológicos. Además, porque el encuentro con lo tecnológico se centra en dar respuesta a preguntas formuladas, como asignación de refuerzo de lo visto en clase. La comunicación se limita a consultas breves a la docente.

Otro factor influyente tal vez es el corto tiempo que dedican los estudiantes al desarrollo de actividades extraclases, puesto que tienen que ayudar a sus padres en las labores agrícolas y no dedican el tiempo necesario para afianzar los conocimientos que han adquirido. Esto se conjuga con el impedimento de no poseer servicio de Internet en sus casas. Ello contribuye a que no se propicie, con mayor fuerza, el desarrollo de las destrezas comunicacionales. En función de promoverlas, hay que entender que ellas, como lo exponen Monsalve-Upegui, Franco-Velásquez, Monsalve-Ríos, Betancur-Trujillo y Ramírez-Salazar (2009) tienen que ver con la cualidad del ser humano de expresarse. $Y$ así indican: 
...la noción de habilidades comunicativas hace referencia a la competencia que tiene una persona para expresar sus ideas, sentimientos, necesidades, sueños y deseos por medio del lenguaje oral y escrito. Asimismo, la capacidad para comprender los mensajes que recibe a través de estos códigos (p. 193).

Esto es, los estudiantes han de comunicarse con sus pares y con el mediador que comparte la construcción de conocimientos a través de los AVA que es el caso estudiado, para promover y fortalecer el dominio del proceso comunicacional y apuntar de ese modo a un aprendizaje significativo. Asimismo, hay que valorar en la investigación que la habilidad de escucha, por parte de los estudiantes, como destreza básica para activar la competencia comunicativa no se ha consolidado. Ello impacta como limitación en el progreso académico del estudiante.

Se asume, como acción positiva, que el trabajo con la tecnología podría ayudar a incrementar las habilidades comunicativas, sin embargo, docentes y estudiantes del cuarto grado de educación primaria, a pesar de contar con la plataforma tecnológica Chamilo poco emplean y desarrollan los AVA, para potenciar las habilidades comunicativas.

\section{Categoría habilidades interpretativas.}

En cuanto al proceso interpretativo se pudo valorar que no se ha desarrollado en el $65 \%$ de los estudiantes. Esto se pudo confirmar, a través de la observación, pues en la actividad de interpretación de textos incluidos para su interpretación, se pudieron evidenciar dificultades para analizar y procesar el contenido del texto; es decir, puede decirse que en ese $65 \%$ anunciado no se dio la interpretación textual. No obstante, hay que decir también que, de manera favorable, el $35 \%$ de los estudiantes observados si desarrolló las habilidades interpretativas.

En la Figura 2, se presentan los porcentajes obtenidos a partir de la observación realizada: 
Figura 2. Categoría: Habilidades interpretativas.

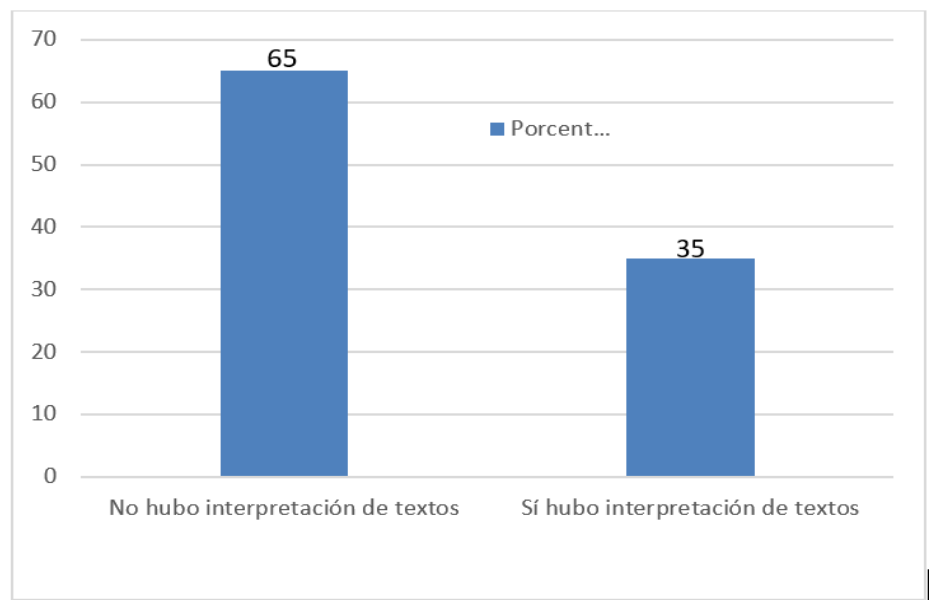

Fuente: elaboración propia

De lo revelado, puede afirmarse que ese $35 \%$, síse considera significativo, puesto que cuando en el futuro inmediato se propongan actividades en los entornos virtuales, aquellos alumnos de grado cuarto de educación primaria que han demostrado fortalezas interpretativas se convertirán en mediadores de sus propios pares en el proceso de entendimiento de los variados textos a los que se enfrenten, dentro del proceso de aprendizaje. $Y$ de ese modo habrá una mejora en la interpretación de los textos digitales que son descargados para realizar las actividades de refuerzo y fortalecimiento del aprendizaje significativo.

Esta acción futura de los aprendices, en cuanto mediadores coetáneos, se vincula con parte de la teoría que ha desarrollado Contreras-Colmenares (2008) y que ha denominado: coetaneidad. Él la definió del siguiente modo:

La coetaneidad concebida y analizada (...) como el atributo que posee el ser humano para apuntalar el aprendizaje de sus semejantes, vale decir, de esos otros seres que se encuentran en el mismo o parecido nivel etario con quienes comparte y aprende (p. 57).

De forma tal que para desarrollar esas habilidades interpretativas han de intervenir diferentes actores y tal propósito se podrá lograr de Prospectiva. Revista de Trabajo Social e intervención social No. 27, enero-junio 2019: pp. 215-240 (c) (1) () () 
manera más espontánea y significativa cuando sean apoyados por sus pares coetáneos. Y cuando otros alumnos tienen mayores fortalezas y habilidades interpretativas el aprendizaje y construcción de conocimientos se hará mucho más significativo y útil. Por ello, es pertinente incluir el planteamiento del Ministerio de Educación de Chile (citado en ArriagadaMena, 2014):

Los lectores competentes extraen y construyen el significado de los textos escritos, no solo a nivel literal sino también a nivel interpretativo. Comprender un texto implica extraer información, inferir o interpretar aspectos que no están expresamente dichos, y evaluarlo críticamente (p. 2).

Promover, fortalecer y conseguir que haya lectores competentes y proficientes es una labor importante que le compete a los maestros; por tanto, una forma de lograrlo es a través de los entornos virtuales de aprendizaje; además, se infiere que con ellos se podrá contribuir a desarrollar con mayor profusión las habilidades interpretativas; éstas también son denominadas por diversos autores como competencias interpretativas. Ellas son experiencias básicas para la comprensión lectora, que podrán ser utilizadas en los textos digitales que se ofrecen con el uso de las TIC y, aparte, también serán desarrolladas y fortalecidas. Dichas competencias o habilidades interpretativas tienen gran significatividad en los procesos de enseñanza y aprendizaje.

\section{Categoría de autoaprendizaje}

Aunque en la encuesta el $60 \%$ de los participantes manifiesta haber encontrado soluciones a dificultades presentadas, como parte de un aprendizaje propio, en la observación se pudo determinar otra situación distinta. Los estudiantes no encontraron estrategias por sí mismos para solucionar los inconvenientes presentados, tanto en los textos como con los recursos tecnológicos.

Necesario, entonces, preponderar el autoaprendizaje. Éste es valorado por la Universidad de Las Américas (2016) como:

...el proceso de enfrentar, de manera cada vez más autónoma y consciente, tus quehaceres académicos y laborales. Es decir, el autoaprendizaje es 
un proceso de construcción del conocimiento que te ayuda a contar con mecanismos de adaptación. En la sociedad actual, las formas de obtener y crear el conocimiento son cada vez más diversas y dinámicas; situación que te desafía a desarrollar la capacidad de comprender por qué, para qué y cuándo necesitas lograr un nuevo conocimiento (p. 1).

Significa que se fundamenta en la construcción de saberes de manera autónoma, que, a su vez, le posibilitarán la adaptación a las circunstancias que se le presenten, en cuanto a las disímiles formas en que se expresa el conocimiento en la sociedad del aprendizaje.

Una posible respuesta a la disparidad encontrada podría estar en que los estudiantes manifiestan no haber trabajado en una computadora que cuente con el servicio de internet; por tanto, no tienen opción para desarrollar actividades que les permitan acceder al conocimiento que se encuentra en los distintos repositorios académicos; sin embargo, se cree necesario que, desde la escuela, se ha de apoyar este proceso de autoaprendizaje. Sobre todo, ha de alentarse a los estudiantes que viven en las zonas rurales, para que puedan superar las limitaciones encontradas que les impiden trabajar con el computador (computadora, portátil, ordenador), en principio, dentro la institución Colegio Integrado Nuestra Señora de la Paz, a través de la Plataforma Chamilo y fuera de la institución.

Ello es conveniente dado que, como refiere Cabero-Almenara (2000) las TIC "condicionan el tipo de aprendizaje a obtener, ya que pueden promover diferentes acciones mentales en los alumnos" (p. 144). Con ello, se puede promover y fortalecer el autoaprendizaje.

\section{Categoría de las habilidades computacionales}

En relación con esta categoría (ver Figura 3), puede decirse que hay disparidad de habilidades entre los estudiantes. Así, por ejemplo, en lo que se refiere al conocimiento de las partes del computador, $60 \%$ de los estudiantes lograron señalar y nombrarlas, $40 \%$ no lo hizo. En lo que se refiere al procedimiento de apagar y encender el computador, $25 \%$ lo encendieron bien y $35 \%$ lo apagó de forma adecuada. 
Figura 3. Categoría: Habilidades computacionales

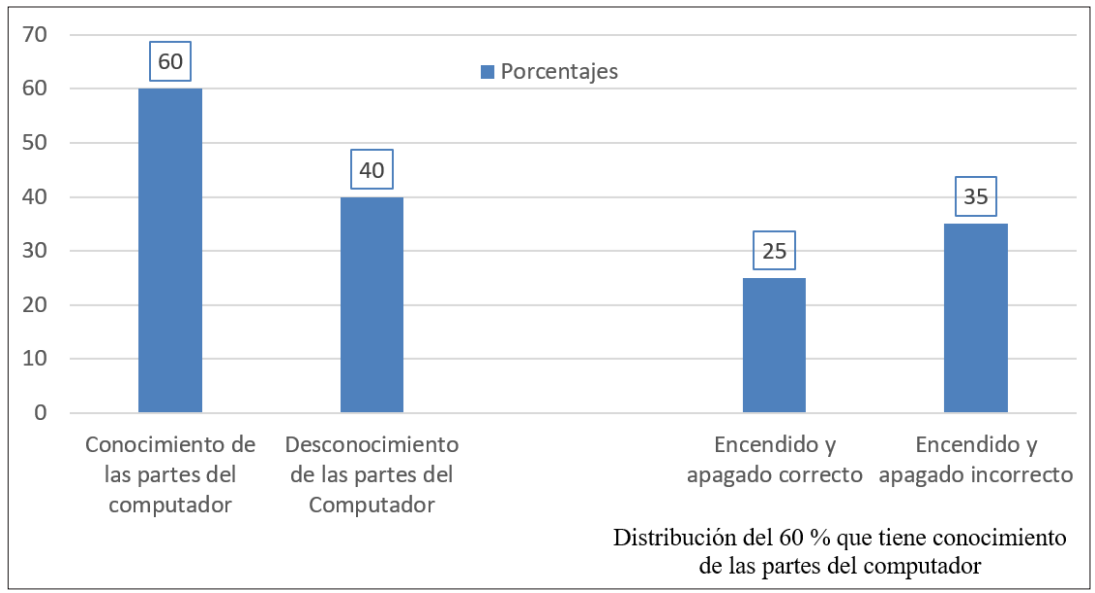

Fuente: elaboración propia

En relación con esta categoría, puede decirse que sí se observó la presencia de dificultad en cuanto al uso del teclado del computador, pues, en su mayoría, no saben usar el teclado para digitar; ello se debe a que no conocen la posición de las letras en el teclado. Se observó también que, al principio, les costaba arrastrar el mouse, así como tampoco seleccionaban de manera adecuada los comandos del menú en los programas utilizados. Se observó que en la medida en que iban teniendo mayor uso de los computadores iban desarrollando las habilidades para dar clic en los botones del mouse; sin embargo, no demostraron el dominio para dar el doble clic; esto pudiera deberse a que no han sido formados en estas funciones o porque aún la psicomotricidad fina aún está en proceso de consolidación dado que son alumnos de cuarto grado.

Se hace necesario indicar que los desempeños sobre conocimiento y manejo del computador, es decir, lo relacionado con las habilidades computacionales que tienen los estudiantes de cuarto grado se encuentran en un rango menor entre los estándares exigidos para los grados de escolaridad que van desde preescolar hasta el tercero de primaria. Esta situación pudiera deberse a que, a pesar de existir unos estándares tecnológicos que indican cuál es la competencia que los estudiantes deben 
alcanzar en cada nivel de estudio, aún los maestros no estamos enseñando los contenidos que se deben impartir en el cuarto grado de primaria.

Habría, en este caso, dos posibles explicaciones que son lucubraciones por cuanto no fue objetivo de la investigación. Una, se desconocen los estándares tecnológicos señalados para cada grado; dos, se le da poca importancia a las clases de informática, lo que se traduce en que se vayan creando dificultades en los estudiantes. Ello, a su vez, les impide tener un buen desempeño en los siguientes grados de escolaridad.

Implica también un reto para los investigadores educativos seguir profundizando con nuevos estudios, para poder revelar cuál es la situación precisa que ocurre al interior de los planteles, con respecto al desarrollo de las habilidades computacionales. Y es elocuente esta situación, puesto que, en este momento, los alumnos de cuarto grado se encuentran en la generación tecnológica denominada: nativos digitales.

Ahora bien, hay que afirmar que, una vez percibida esta situación, se encontró también una disparidad en cuanto a las respuestas dadas por los estudiantes, que fueron registradas en el cuestionario. Es así que $95 \%$ manifiesta que les gustaría seguir trabajando en la plataforma, pues les facilita la concentración que los conduce a un mejoramiento importante en el rendimiento académico. Dato que se ratifica con las respuestas de un $100 \%$ de ellos que considera que estas actividades les ayudan a mejorar el rendimiento académico.

Una interpretación a esta contradicción evidenciada se centraría en establecer que $80 \%$ manifiesta haber tenido algún problema con la plataforma especialmente en la exploración de las diferentes secciones; mientras que $65 \%$ dice no haber desarrollado actividades de aprendizaje en la computadora con apoyo en internet (ver Figura 4). 
Figura 4. Categoría: Opiniones respecto al uso de la tecnología

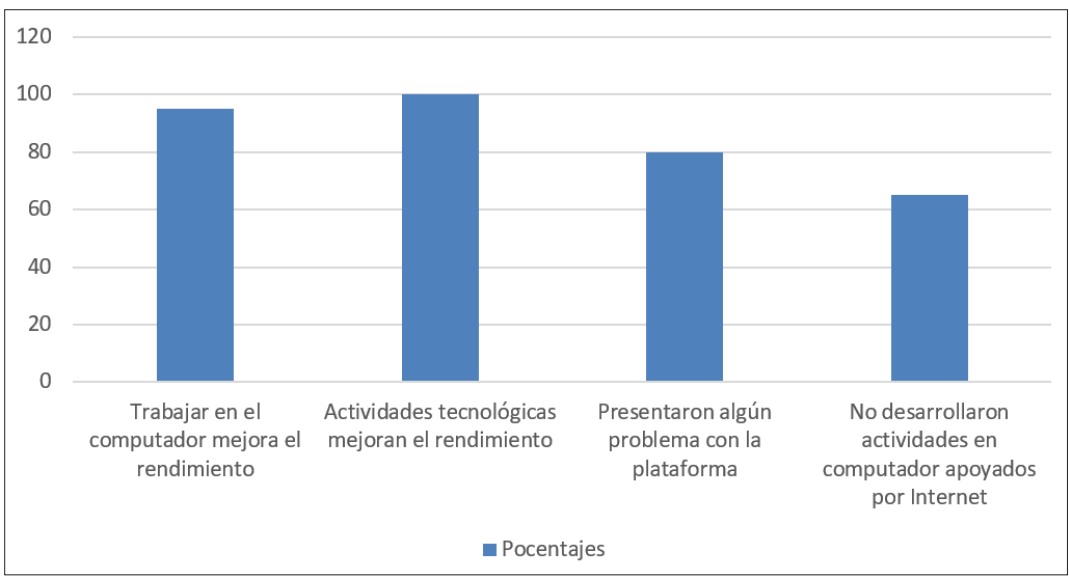

Fuente: elaboración propia

Con base en estas informaciones se valora que, en cuanto al uso de los AVA siguen existiendo, por parte de los estudiantes del grado cuarto de primaria, las dificultades en su uso, debido al poco dominio que tienen con las TIC. Es, entonces, imperativo que se desarrollen actividades de concienciación entre el profesorado, para que se aproxime a un mayor y mejor aprovechamiento de la plataforma Chamilo.

Se da por concluido el proceso de análisis a la información recabada, que necesariamente, evidencia la necesidad de seguir profundizando con nuevas investigaciones, acerca de la temática expuesta.

\section{Conclusiones}

Realizado todo el transitar investigativo se considera oportuno dar a conocer algunas informaciones a modo de conclusiones en los siguientes términos:

- Los AVA pueden convertirse en espacios de formación integral de los estudiantes de cuarto grado con la asunción de un compromiso decidido por parte de los docentes. Hasta ahora, su incorporación ha estado limitada por varios factores, tanto de carácter personal, como 
tecnológico. En lo primero por desconocimiento de la importancia de su inclusión en las aulas de clase para mejorar la práctica educativa, que conduzca a lograr aprendizajes significativos.

- Los AVA, como aplicación informática, no suponen en sí mismas un desarrollo del potencial pedagógico de estas tecnologías, sino que las enmarcan en los modelos unidireccionales de la educación tradicional; por tanto, la plataforma Chamilo se considera como un recurso tecnológico que viene a apoyar y a enriquecer los procesos de enseñanza aprendizaje, y no como un todo que viene a solucionar las dificultades de aprendizaje que afectan actualmente a la educación.

- Sobre la base de valorar que estamos inmersos en un mundo digital, se hace necesario incluir, en nuestra práctica educativa, estrategias que permitan a los estudiantes desarrollar habilidades que ayuden a desenvolverse en la sociedad de la información. Así como el desarrollo de otras habilidades que han de formar parte de la formación integral de los estudiantes.

- Para desarrollar actividades de aprendizaje en la plataforma Chamilo se necesita que los estudiantes entiendan y comprendan cada uno de los pasos que se llevarán a cabo en el trabajo que se va a realizar; además se ha de tener conocimiento sobre la manera cómo se utiliza dicho recurso tecnológico.

- Los estudiantes de cuarto grado de primaria no poseen habilidades de autoaprendizaje para trabajar en el desarrollo de actividades en un ambiente virtual y, además, son poco metódicos en la búsqueda de estrategias que contribuyan a la solución de inconvenientes presentados. No obstante, los estudiantes que han desarrollado ciertas habilidades se convierten en mediadores de sus pares, cuando hacen preguntas, despejan inquietudes y encuentran opciones alternas para cumplir con las tareas asignadas.

- Para que los estudiantes puedan manejar adecuadamente los AVA deben tener conocimiento de la tecnología de la información y 
la comunicación. Por tanto se requiere tener dominio de dicha aplicación informática.

- Se pudo valorar que los estudiantes que han tenido la oportunidad de interactuar con el computador son más efectivos en el uso de dichos espacios virtuales y, por ende, su desempeño personal en el progreso académico es más significativo. Contrario en aquellos que, en pocas ocasiones, han trabajado con estos recursos tecnológicos, pues se les hace mucho más difícil el acceso a la virtualidad, lo que conduce, en ocasiones a cierta frustración que ha de ser atendida por los docentes.

\section{Referencias bibliográficas}

Arias-Arranz, F.M. (2017). Entornos virtuales de aprendizaje en los centros de educación infantil y primaria. (Tesis de pregrado). Universidad de Valladolid. Facultad de Educación de Segovia, Segovia, España.

Arriagada-Mena, D.E. (2014). Desarrollo de habilidades de comprensión lectora, mediante la integración de tablet. (Tesis de maestría). Universidad de Chile. Facultad de Ciencias Sociales Departamento de Educación. Maestría en Educación Mención Informática Educativa.

Cabero-Almenara, J. (2000) Nuevas tecnologías aplicadas a la educación. Madrid: Síntesis.

Castañeda-Castañeda, A., Carrilo-Álvarez, J. y Quintero-Monreal, Z.Z. (2013). El uso de las TIC en Educación Primaria: la Experiencia ENCICLOMEDIA. México: Red de Investigadores Educativos, A. C.

Cebrián de la Serna, M. (Coord.) (2003). Enseñanza virtual para la innovación universitaria. Madrid: Narcea.

Contreras-Colmenares, A.F. (2004). Mediación de procesos cognitivos y aprendizaje de la lectura. Venezuela: Litoformas.

Contreras-Colmenares, A. (2008). La coetaneidad en el proceso de mediación y aprendizaje de la lectura. Acción Pedagógica, 17(1), 56-67.

Daniels, H. (2003). Vygotsky y la Pedagogía. España: Paidós.

García-Salinero, J. (2004). Estudios descriptivos. Nure Investigación, (7). Recuperado de http://www.nureinvestigacion.es/OJS/index.php/nure/article/ view/180/163.

Gros-Salvat, B. (Ed.). (2011). Evolución y retos de la educación virtual. Construyendo el E-learning del siglo XXI. España: Editorial UOC. 
Hernández, R., Fernández, C. y Baptista, P. (1994). Metodología de la investigación (4a. ed.). México MacGraw Hill.

Hernández, R. Fernández, C. y Baptista, P. (2010). Metodología de la investigación (5a. ed.). México: McGraw Hill.

Leiva-Zea, F. (2010). Investigación Científica. Quito: Libresa.

Monsalve-Upegui, M., Franco-Velásquez, M., Monsalve-Ríos, M., BetancurTrujillo, V. y Ramírez-Salazar, D. (2009). Desarrollo de las habilidades comunicativas en la escuela nueva. Revista Educación y Pedagogía, 21(55), 189-210.

Ospina-Pineda, D. (2014). ¿Qué es un ambiente virtual de aprendizaje? Recuperado de http://aprendeenlinea.udea.edu.co/boa/contenidos.php/ cee1c4c4045aded3a9cecfbcdaf9d8db/144/1/contenido/.

Romera-Blasco, C.M. (2014). Análisis del impacto de las TIC en educación primaria y pautas de intervención para su utilización óptima y eficiente. (Tesis de pregrado). Universidad Internacional de La Rioja. Facultad de Educación. Soria, España.

Rubio-Moraga, Á. (2003). Internet y enseñanza: la educación virtual. En C. Barrera del Barrio, M. García-López y F. Martínez-Vallvey (Eds.) La comunicación: industria, conocimiento, profesión (pp.1-13). España: Edipo.

Silva, J. (2017). Un modelo pedagógico virtual centrado en las E-actividades. RED. Revista de Educación a Distancia, (53), 1-20. doi: 10.6018/red/53/10.

Universidad de Las Américas (2016). Fichas para el estudiante. Autoaprendizaje. Recuperado de http://www.udla.cl/portales/tp6c6191b55q52/uploadImg/File/ autoaprendizaje/Ficha\%200\%20Autoaprendizaje.pdf.

Valencia-Vallejo, N.G., Huertas-Bustos, A.P. y Baracaldo-Ramírez, P.O. (2014). Los ambientes virtuales de aprendizaje: una revisión de publicaciones entre 2003 y 2013, desde la perspectiva de la pedagogía basada en la evidencia. Revista Colombiana de Educación, (66), 73-103.

Villar, L.M. \& Marcelo, C. (1992). Kombination Qualitativer und Quantitativer Methoden. En G.L. Huber (ed.). Qualitative Analyse. Computereinsatz in der Sozialforschung (pp. 177-218). Munich: Oldenbourg.

Cómo citar:

Contreras-Colmenares, A.F y Garcés-Díaz, L.M. (2019). Ambientes Virtuales de Aprendizaje: dificultades de uso en los estudiantes de cuarto grado. Prospectiva. Revista de Trabajo Social e intervención social, (27), 215-240. doi: 10.25100/ prts.v0i27.7273. 